Supporting Information for

\title{
A Comprehensive Model for the Electronic Structures of 1,2,4-Cyclohexatriene and Related Compounds
}

Patrick W. Musch, Daniel Scheidel, and Bernd Engels*

Institut für Organische Chemie, Universität Würzburg, Am Hubland. D-97074 Würzburg (Germany)

$$
\text { Fax: (+49) } 9318885331
$$

engels@chemie.uni-wuerzburg.de 
Table 1 Alsolute energies in a.u., $\left\langle S^{2}\right\rangle$ and imaginary frequences

\begin{tabular}{|c|c|c|c|c|c|c|c|}
\hline & B3LYP & $\mathrm{MRCl}$ & CASPT2 & $\operatorname{CcSD}(T)$ & $\Delta G_{298}^{\text {corr }}$ & $\left\langle S^{2}\right\rangle$ & Imag. Freq. $\left(\mathrm{cm}^{-1}\right)$ \\
\hline $1 a$ & -218.29791 & -217.6838 & -217.657613 & -217.84152 & 0.052924 & & \\
\hline $1 b$ & -218.27854 & -217.6646 & -217.639384 & & 0.051735 & 1.0416 & -544 \\
\hline $1 d$ & -218.29547 & -217.6797 & -217.654676 & -217.838105 & 0.051811 & & \\
\hline $1^{2-} b$ & -218.09210 & -217.5662 & -217.551535 & & 0.043346 & 1.0161 & -504 \\
\hline $1^{2-} \mathrm{c}$ & -218.18034 & -217.5916 & -217.578046 & & 0.049459 & & \\
\hline $2 a$ & -232.12975 & -231.5087 & -231.483800 & -231.67254 & 0.068345 & & \\
\hline $2 b$ & -232.12359 & -231.4914 & -231.466183 & & 0.066315 & 1.0594 & -215 \\
\hline $2 c$ & -232.09730 & -231.4667 & -231.434814 & -231.629949 & 0.07197 & & \\
\hline $27 b$ & -231.51942 & -230.8884 & -230.882618 & & 0.05219 & 1.0221 & \\
\hline $2 \mathrm{c}$ & -231.59640 & -230.9690 & -230.957735 & & 0.057206 & & \\
\hline $3 b$ & -248.16809 & -247.5170 & -247.504089 & & 0.057445 & 1.0360 & -212 \\
\hline $3 c$ & -248.20478 & -247.5572 & -247.540560 & & 0.059112 & & \\
\hline $3^{+} a$ & -248.50463 & -247.8643 & -247.845950 & -248.038744 & 0.071098 & & \\
\hline $3^{+} b$ & -248.49761 & -247.8500 & -247.827383 & & 0.069249 & 1.0607 & -166 \\
\hline $3^{+} \mathrm{c}$ & -248.45985 & -247.8033 & -247.784587 & -247.983391 & 0.071174 & & \\
\hline $4 a$ & -268.04714 & -267.3805 & -267.367063 & -267.56104 & 0.046259 & & \\
\hline $4 b$ & -268.02347 & -267.3534 & -267.345488 & & 0.048183 & 1.0449 & \\
\hline $4 c$ & -268.04544 & -267.3749 & -267.360815 & -267.558014 & 0.046171 & & -251 \\
\hline $5 a$ & -435.89359 & -434.9089 & -434.882448 & -435.063723 & 0.045158 & & \\
\hline $5 b$ & -435.86619 & -434.8788 & -434.855248 & & 0.043326 & 1.0476 & -756 \\
\hline $5 d$ & -435.88411 & -434.8967 & -434.870203 & -435.051829 & 0.043773 & & -596 \\
\hline $6 a$ & -483.54929 & -482.5549 & -482.520712 & -482.715546 & 0.056109 & & \\
\hline $6 b$ & -483.53064 & -482.5329 & -482.499501 & & 0.054169 & 1.0573 & -291 \\
\hline $6 c$ & -483.51277 & -482.5076 & -482.475275 & -482.671514 & 0.052771 & & -1334 \\
\hline $6 d$ & -483.47214 & & & & 0.055699 & & \\
\hline $7 a$ & -534.79711 & -533.7785 & & -533.954044 & 0.049092 & & \\
\hline $7 b$ & -534.78202 & -533.7570 & & & 0.047077 & 1.0909 & -235 \\
\hline $7 c$ & -534.77296 & -533.7506 & & -533.929066 & 0.047284 & & \\
\hline $8 a$ & -837.46037 & -836.0531 & & -836.237247 & 0.032131 & & \\
\hline
\end{tabular}




\begin{tabular}{|c|c|c|c|c|c|c|c|}
\hline $8 b$ & -837.43319 & -836.0169 & & & 0.029958 & 0.9668 & -311 \\
\hline $8 c$ & -837.42292 & -836.0183 & & -836.199886 & 0.030154 & & \\
\hline $9 a$ & -591.04306 & -590.0079 & -589.984812 & -590.191967 & 0.041582 & & \\
\hline $9 b$ & -591.02568 & -589.9840 & -589.964944 & & 0.03927 & 1.0452 & -741 \\
\hline $9 c$ & -591.03223 & -589.9915 & -589.971802 & -590.175527 & 0.04129 & & -432 \\
\hline
\end{tabular}

Table 2 Energies and characters of the electronic states of 1,2,4-cyclohexatriene and its hetero analogues as indicated in Scheme1. ${ }^{\text {a) }}$

\begin{tabular}{|c|c|c|c|c|c|c|c|}
\hline & \multicolumn{2}{|c|}{$B 3 L Y P^{b}$} & \multicolumn{2}{|c|}{ CASPT2 $^{C}$} & \multicolumn{2}{|c|}{$M R C I+Q^{d}$} & $\operatorname{CCSD}(T)^{e}$ \\
\hline$x$ & diradical & zwitterion & diradical & zwitterion & diradical & zwitterion & zwitterion \\
\hline $\mathrm{BH}(1)^{f}$ & +11 & +1 & +11 & +1 & +11 & +2 & +1 \\
\hline $\mathrm{CH}_{2}(2)^{\mathrm{g}}$ & +3 & +23 & +10 & +33 & +10 & +29 & +29 \\
\hline $\mathrm{NH}(3)^{\mathrm{g}, \mathrm{h}}$ & +22 & 0 & +22 & 0 & +24 & $0^{g}$ & n.a. ${ }^{h}$ \\
\hline$O(4)^{g}$ & +16 & +1 & +15 & +4 & +18 & +3 & +2 \\
\hline $\operatorname{AlH}(5)^{f}$ & +16 & +5 & +16 & +7 & +18 & +7 & +7 \\
\hline $\mathrm{SiH}_{2}(\mathbf{6})^{\mathrm{f}}$ & +10 & $+21^{i}$ & +12 & +26 & +13 & +28 & +26 \\
\hline $\mathrm{PH}(7)^{\mathrm{g}}$ & +8 & +14 & n.a. & n.a. & +12 & +16 & +15 \\
\hline $\mathrm{PH}(\mathbf{8})^{\mathrm{g}}$ & +16 & +22 & n.a. & n.a. & +21 & +21 & +22 \\
\hline$S(9)^{g}$ & +9 & +7 & +11 & +8 & +14 & +10 & +10 \\
\hline
\end{tabular}

a) All values are given in $\mathrm{kcal} \cdot \mathrm{mol}^{-1}$ with respect to the allene structure except for 3 .

b) (U)B3LYP/cc-pVDZ//(U)B3LYP/cc-pVDZ

c) CASPT2/ano-I//(U)B3LYP/cc-pVDZ

d) $\mathrm{MRCI}+\mathrm{Q} / \mathrm{ano}-\mathrm{I} / /(\mathrm{U}) \mathrm{B} 3 \mathrm{LYP} / \mathrm{cc}-\mathrm{pVDZ}$

e) $\operatorname{CCSD}(T) / c c-p V T Z(f / p) / / B 3 L Y P / c c-p V D Z$

f) zwitterion of type d (see Scheme 1)

g) zwitterion of type c (see Scheme 1)

h) zwitterion is ground state

i) zwitterion c $48 \mathrm{kcal} \cdot \mathrm{mol}^{-1}$ higher than allene a 
Table 3 Absolute orbital and single determinant energies

\begin{tabular}{|c|c|c|c|c|c|c|c|c|}
\hline & \multicolumn{2}{|c|}{$\mathrm{X}=\mathrm{CH}_{2}$} & \multicolumn{2}{|c|}{$\mathrm{X}=\mathrm{CH}^{-}$} & \multicolumn{2}{|c|}{$\mathrm{X}=\mathrm{NH}$} & \multicolumn{2}{|c|}{$\mathrm{X}=\mathrm{O}$} \\
\hline & $2 b$ & 2c & $2 b^{-}$ & $2 c^{-}$ & $3 b$ & $3 c$ & $4 b$ & $4 c$ \\
\hline$\varepsilon_{\pi}^{*}$ & -0.091 & -0.076 & +0.207 & +0.230 & -0.038 & -0.018 & -0.073 & -0.056 \\
\hline$\varepsilon_{\sigma}$ & -0.132 & -0.175 & +0.073 & +0.028 & -0.134 & -0.172 & -0156 & -0.194 \\
\hline$E_{\sigma \pi}^{\operatorname{det}{ }^{*}}(\mathbf{b})$ & \multicolumn{2}{|c|}{-230.580} & \multicolumn{2}{|c|}{-229.964} & \multicolumn{2}{|c|}{-246.576} & \multicolumn{2}{|c|}{-266.392} \\
\hline $\mathrm{E}_{\sigma \pi}^{\operatorname{det}}(\mathbf{c})$ & \multicolumn{2}{|c|}{-230.553} & \multicolumn{2}{|c|}{-229.934} & \multicolumn{2}{|c|}{-246.554} & \multicolumn{2}{|c|}{-266.372} \\
\hline $\mathrm{E}_{\sigma}^{\mathrm{det}}{ }^{2}(\mathbf{c})$ & \multicolumn{2}{|c|}{-230.548} & \multicolumn{2}{|c|}{-230.046} & \multicolumn{2}{|c|}{-246.611} & \multicolumn{2}{|c|}{-266.409} \\
\hline
\end{tabular}

\section{Geometries optimized with (U)B3LYP/cc-pVDZ in cartesian coordinates}

The labelling is according to the manuscript (Figure 1 and Scheme 1)

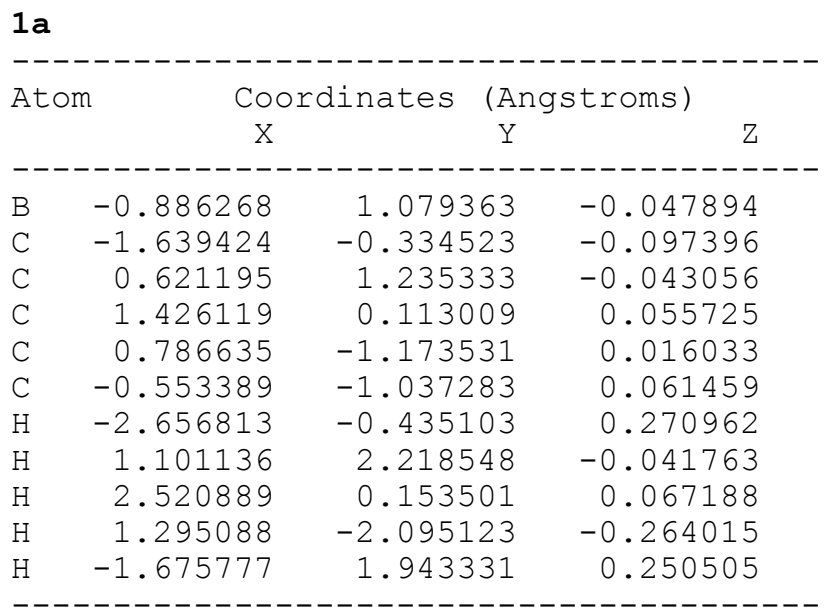

\section{$1 b$}

\begin{tabular}{|c|c|c|c|}
\hline Atom & $\begin{array}{l}\text { Coord } \\
X\end{array}$ & $\begin{array}{c}\text { rates (An } \\
\text { Y }\end{array}$ & roms ) \\
\hline B & -1.308355 & 0.000000 & 0.726272 \\
\hline $\mathrm{C}$ & -1.313159 & 0.000000 & -0.838385 \\
\hline $\mathrm{C}$ & 0.088425 & 0.000000 & 1.411223 \\
\hline $\mathrm{C}$ & 1.240108 & 0.000000 & 0.651302 \\
\hline $\mathrm{C}$ & 1.166893 & 0.000000 & -0.775414 \\
\hline $\mathrm{C}$ & -0.085585 & 0.000000 & -1.413450 \\
\hline $\mathrm{H}$ & -2.225437 & 0.000000 & -1.445670 \\
\hline $\mathrm{H}$ & 0.200311 & 0.000000 & 2.500814 \\
\hline $\mathrm{H}$ & 2.234536 & 0.000000 & 1.110581 \\
\hline $\mathrm{H}$ & 2.089195 & 0.000000 & -1.364416 \\
\hline $\mathrm{H}$ & -2.336916 & 0.000000 & 1.355673 \\
\hline
\end{tabular}


1c

\begin{tabular}{|c|c|c|c|}
\hline Atom & $\begin{array}{l}\text { Coorc } \\
\mathrm{X}\end{array}$ & $\begin{array}{c}\text { nates (Ang } \\
\text { Y }\end{array}$ & ms ) \\
\hline B & 1.284094 & -0.544235 & 0.000000 \\
\hline $\mathrm{C}$ & 1.285942 & 1.077456 & 0.000000 \\
\hline $\mathrm{C}$ & 0.043817 & -1.382730 & 0.000000 \\
\hline $\mathrm{C}$ & -1.204124 & -0.764012 & 0.000000 \\
\hline $\mathrm{C}$ & -1.261574 & 0.659164 & 0.000000 \\
\hline C & 0.000000 & 1.157249 & 0.000000 \\
\hline $\mathrm{H}$ & 2.192297 & 1.663231 & 0.000000 \\
\hline $\mathrm{H}$ & 0.080878 & -2.475333 & 0.000000 \\
\hline $\mathrm{H}$ & -2.150619 & -1.315244 & 0.000000 \\
\hline $\mathrm{H}$ & -2.165643 & 1.261855 & 0.000000 \\
\hline $\mathrm{H}$ & 2.438252 & -0.896095 & 0.000000 \\
\hline
\end{tabular}

\section{$1^{2-} b$}

\begin{tabular}{|c|c|c|c|}
\hline Atom & $\begin{array}{c}\text { Coord } \\
\mathrm{X}\end{array}$ & Coordinates (Angstroms) & oms ) \\
\hline B & 1.338722 & -0.699942 & 0.000000 \\
\hline $\mathrm{C}$ & 1.306102 & 0.893761 & 0.000000 \\
\hline $\mathrm{C}$ & 0.022065 & -1.416347 & 0.000000 \\
\hline $\mathrm{C}$ & -1.254368 & -0.709603 & 0.000000 \\
\hline $\mathrm{C}$ & -1.219638 & 0.763831 & 0.000000 \\
\hline $\mathrm{C}$ & 0.000000 & 1.412010 & 0.000000 \\
\hline $\mathrm{H}$ & 2.186038 & 1.567083 & 0.000000 \\
\hline $\mathrm{H}$ & -0.044404 & -2.528066 & 0.000000 \\
\hline $\mathrm{H}$ & -2.232291 & -1.229845 & 0.000000 \\
\hline $\mathrm{H}$ & -2.169028 & 1.336814 & 0.000000 \\
\hline $\mathrm{H}$ & 2.441110 & -1.308192 & 0.000000 \\
\hline
\end{tabular}

\section{$1^{2-} \mathrm{C}$}

$\begin{array}{llll}\text { Atom } & \text { Coordinates } & \text { (Angstroms) } & \\ & \mathrm{X} & \mathrm{Y} & \mathrm{Z}\end{array}$

$\begin{array}{lrrr}- & -1.274348 & -0.795756 & 0.000000 \\ \text { B } & -0.097841 & -1.459477 & 0.000000 \\ \text { C } & 1.259210 & 0.726488 & 0.000000 \\ \text { C } & 0.000000 & 1.362856 & 0.000000 \\ \text { C } & -1.213922 & 0.637462 & 0.000000 \\ \text { C } & -1.371599 & -0.790840 & 0.000000 \\ \text { H } & -0.152151 & -2.584646 & 0.000000 \\ \text { H } & 2.155955 & 1.383288 & 0.000000 \\ \text { H } & -0.057277 & 2.480187 & 0.000000 \\ \text { H } & -2.133757 & 1.278045 & 0.000000 \\ \text { H } & 2.360397 & -1.437032 & 0.000000 \\ -\end{array}$


$2 a$

\begin{tabular}{|c|c|c|c|}
\hline$n$ & $\begin{array}{c}\text { Coor } \\
X\end{array}$ & \multicolumn{2}{|c|}{ Coordinates (Angstroms) } \\
\hline $\mathrm{C}$ & 1.155061 & -0.867191 & -0.182352 \\
\hline $\mathrm{C}$ & 1.253229 & 0.588107 & 0.245748 \\
\hline $\mathrm{C}$ & -0.067246 & 1.301714 & -0.056757 \\
\hline $\mathrm{C}$ & -1.241503 & 0.651232 & -0.215865 \\
\hline $\mathrm{C}$ & -1.244852 & -0.782199 & 0.166455 \\
\hline $\mathrm{C}$ & -0.064392 & -1.380819 & -0.023237 \\
\hline $\mathrm{H}$ & -2.167849 & 1.193697 & -0.424222 \\
\hline $\mathrm{H}$ & 2.061198 & 1.048669 & -0.349165 \\
\hline $\mathrm{H}$ & 1.530430 & 0.783547 & 1.302738 \\
\hline $\mathrm{H}$ & -0.037176 & 2.395323 & -0.097915 \\
\hline . & -2.060769 & -1.179225 & 0.778740 \\
\hline & 1.932392 & -1.307078 & -0.814134 \\
\hline
\end{tabular}

\section{$2 b$}

\begin{tabular}{|c|c|c|c|}
\hline tom & $\begin{array}{l}\text { Coorc } \\
x\end{array}$ & $\begin{array}{c}\text { rates (Angs } \\
\text { Y }\end{array}$ & oms ) \\
\hline $\mathrm{C}$ & -1.128601 & -0.928476 & 0.000000 \\
\hline C & -1.303431 & 0.579814 & 0.000000 \\
\hline $\mathrm{C}$ & 0.000000 & 1.330493 & 0.000000 \\
\hline $\mathrm{C}$ & 1.219532 & 0.709195 & 0.000000 \\
\hline $\mathrm{C}$ & 1.332205 & -0.718345 & 0.000000 \\
\hline $\mathrm{C}$ & 0.126357 & -1.429664 & 0.000000 \\
\hline $\mathrm{H}$ & 2.133305 & 1.311200 & 0.000000 \\
\hline $\mathrm{H}$ & -1.921064 & 0.887559 & 0.873073 \\
\hline $\mathrm{H}$ & -1.921064 & 0.887559 & -0.873073 \\
\hline $\mathrm{H}$ & -0.051727 & 2.422896 & 0.000000 \\
\hline $\mathrm{H}$ & 2.307036 & -1.208849 & 0.000000 \\
\hline $\mathrm{H}$ & -2.022859 & -1.558468 & 0.000000 \\
\hline
\end{tabular}

2c

\begin{tabular}{|c|c|c|c|}
\hline Atom & $\begin{array}{l}\text { Coorc } \\
X\end{array}$ & $\begin{array}{c}\text { lates } \\
\text { Y Angs }\end{array}$ & coms) \\
\hline $\mathrm{C}$ & -1.266734 & 0.587128 & 0.000000 \\
\hline $\mathrm{C}$ & 0.000000 & 1.372468 & 0.000000 \\
\hline $\mathrm{C}$ & 1.253385 & 0.591704 & 0.000000 \\
\hline $\mathrm{C}$ & 1.179982 & -0.767855 & 0.000000 \\
\hline $\mathrm{C}$ & -0.109651 & -1.410534 & 0.000000 \\
\hline C & -1.401330 & -0.807227 & 0.000000 \\
\hline $\mathrm{H}$ & 2.091230 & -1.374161 & 0.000000 \\
\hline $\mathrm{H}$ & -0.004356 & 2.075348 & 0.863015 \\
\hline $\mathrm{H}$ & -0.004356 & 2.075348 & -0.863015 \\
\hline $\mathrm{H}$ & 2.211464 & 1.119160 & 0.000000 \\
\hline 11 & -0.059688 & -2.512170 & 0.000000 \\
\hline 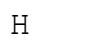 & -2.168206 & 1.222365 & 0.000000 \\
\hline
\end{tabular}


$2^{-} \mathrm{b}$

\begin{tabular}{|c|c|c|c|}
\hline Atom & $\begin{array}{l}\text { Coord } \\
\mathrm{X}\end{array}$ & $\begin{array}{c}\text { nates (An } \\
\text { Y }\end{array}$ & $n s$ ) \\
\hline C & 0.000000 & -1.405502 & 0.000000 \\
\hline C & 1.246329 & -0.808910 & 0.000000 \\
\hline a & 1.234525 & 0.668224 & 0.000000 \\
\hline $\mathrm{C}$ & 0.000000 & 1.329166 & 0.000000 \\
\hline $\mathrm{C}$ & -1.234532 & 0.668227 & 0.000000 \\
\hline $\mathrm{C}$ & -1.246323 & -0.808910 & 0.000000 \\
\hline $\mathrm{H}$ & 2.185084 & -1.372477 & 0.000000 \\
\hline $\mathrm{H}$ & 2.175606 & 1.230514 & 0.000000 \\
\hline 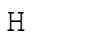 & 0.000002 & 2.430169 & 0.000000 \\
\hline 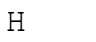 & -2.175610 & 1.230511 & 0.000000 \\
\hline $\mathrm{H}$ & -2.185077 & -1.372481 & 0.000000 \\
\hline
\end{tabular}

$2^{-} \mathrm{c}$

\begin{tabular}{|c|c|c|c|}
\hline Atom & $\begin{array}{l}\text { Coord } \\
x\end{array}$ & $\begin{array}{c}\text { nates (Angs } \\
\text { Y }\end{array}$ & oms ) \\
\hline $\mathrm{C}$ & -1.375598 & -0.746792 & 0.000000 \\
\hline $\mathrm{C}$ & -1.222473 & 0.671547 & 0.000000 \\
\hline $\mathrm{C}$ & 0.000000 & 1.366893 & 0.000000 \\
\hline $\mathrm{C}$ & 1.208001 & 0.655800 & 0.000000 \\
\hline $\mathrm{C}$ & 1.146312 & -0.744581 & 0.000000 \\
\hline $\mathrm{C}$ & -0.102736 & -1.390972 & 0.000000 \\
\hline $\mathrm{H}$ & -2.130854 & 1.306068 & 0.000000 \\
\hline $\mathrm{H}$ & 0.021129 & 2.469027 & 0.000000 \\
\hline $\mathrm{H}$ & 2.172185 & 1.179267 & 0.000000 \\
\hline $\mathrm{H}$ & 2.082032 & -1.327339 & 0.000000 \\
\hline $\mathrm{H}$ & -0.065530 & -2.498390 & 0.000000 \\
\hline
\end{tabular}

\section{$3 b$}

\begin{tabular}{|c|c|c|c|}
\hline Atom & $\begin{array}{l}\text { Cooro } \\
X\end{array}$ & $\begin{array}{c}\text { nates (Angs } \\
\text { Y }\end{array}$ & oms ) \\
\hline $\mathrm{N}$ & -1.195186 & 0.590322 & 0.000000 \\
\hline $\mathrm{C}$ & 0.000000 & 1.303971 & 0.000000 \\
\hline $\mathrm{C}$ & -1.172889 & -0.826900 & 0.000000 \\
\hline C & 0.054306 & -1.409658 & 0.000000 \\
\hline C & 1.294263 & -0.774007 & 0.000000 \\
\hline C & 1.208311 & 0.666619 & 0.000000 \\
\hline $\mathrm{H}$ & -2.077471 & 1.081729 & 0.000000 \\
\hline $\mathrm{H}$ & -0.095760 & 2.389679 & 0.000000 \\
\hline $\mathrm{H}$ & 2.118388 & 1.271030 & 0.000000 \\
\hline $\mathrm{H}$ & 2.250392 & -1.295276 & 0.000000 \\
\hline $\mathrm{H}$ & -2.133191 & -1.339571 & 0.000000 \\
\hline
\end{tabular}


$3 c$

\begin{tabular}{|c|c|c|c|}
\hline Atom & $\begin{array}{l}\text { Coord } \\
X\end{array}$ & $\begin{array}{c}\text { lates (Angs } \\
\text { Y }\end{array}$ & oms ) \\
\hline $\mathrm{N}$ & 0.000000 & 1.283999 & 0.000000 \\
\hline $\mathrm{C}$ & -1.207198 & 0.635380 & 0.000000 \\
\hline $\mathrm{C}$ & -1.363749 & -0.756171 & 0.000000 \\
\hline $\mathrm{C}$ & -0.101130 & -1.411836 & 0.000000 \\
\hline $\mathrm{C}$ & 1.146888 & -0.761468 & 0.000000 \\
\hline $\mathrm{C}$ & 1.179447 & 0.622741 & 0.000000 \\
\hline $\mathrm{H}$ & 0.019843 & 2.303612 & 0.000000 \\
\hline $\mathrm{H}$ & 2.088928 & 1.222718 & 0.000000 \\
\hline $\mathrm{H}$ & 2.091983 & -1.312148 & 0.000000 \\
\hline $\mathrm{H}$ & -0.074735 & -2.511451 & 0.000000 \\
\hline $\mathrm{H}$ & -2.051572 & 1.337404 & 0.000000 \\
\hline
\end{tabular}

\section{$3^{+} a$}

\begin{tabular}{|c|c|c|c|}
\hline & $\begin{array}{l}\text { Coordinates } \\
\text { X }\end{array}$ & $\begin{array}{c}\text { rates (Angs } \\
\text { Y }\end{array}$ & coms) \\
\hline $\mathrm{N}$ & 1.176734 & 0.543192 & 0.268098 \\
\hline $\mathrm{C}$ & -1.249552 & -0.784662 & 0.201402 \\
\hline $\mathrm{C}$ & -0.088687 & -1.376567 & -0.068943 \\
\hline $\mathrm{C}$ & 1.123044 & -0.879316 & -0.221801 \\
\hline $\mathrm{C}$ & -0.094678 & 1.294430 & -0.084207 \\
\hline $\mathrm{C}$ & -1.255083 & 0.636105 & -0.230533 \\
\hline $\mathrm{H}$ & 1.972753 & -1.202035 & -0.822057 \\
\hline $\mathrm{H}$ & 1.353361 & 0.640066 & 1.285840 \\
\hline $\mathrm{H}$ & 0.045172 & 2.374620 & -0.135333 \\
\hline $\mathrm{H}$ & -2.166379 & 1.189375 & -0.469425 \\
\hline $\mathrm{H}$ & -2.024090 & -1.156160 & 0.878866 \\
\hline 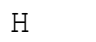 & 1.971778 & 1.011846 & -0.190089 \\
\hline
\end{tabular}

$3^{+} b$

\begin{tabular}{|c|c|c|c|}
\hline Atom & $\begin{array}{l}\text { Coorc } \\
X\end{array}$ & $\begin{array}{c}\text { nates (Ang } \\
\text { Y }\end{array}$ & coms ) \\
\hline $\mathrm{N}$ & -1.253327 & 0.523578 & 0.000000 \\
\hline $\mathrm{C}$ & 1.376187 & -0.702368 & 0.000000 \\
\hline $\mathrm{C}$ & 0.180464 & -1.423118 & 0.000000 \\
\hline C & -1.081276 & -0.969602 & 0.000000 \\
\hline $\mathrm{C}$ & 0.000000 & 1.322984 & 0.000000 \\
\hline $\mathrm{C}$ & 1.218018 & 0.719196 & 0.000000 \\
\hline $\mathrm{H}$ & -2.014487 & -1.531961 & 0.000000 \\
\hline $\mathrm{H}$ & 2.099482 & 1.365247 & 0.000000 \\
\hline $\mathrm{H}$ & 2.360675 & -1.171136 & 0.000000 \\
\hline $\mathrm{H}$ & -0.174322 & 2.398026 & 0.000000 \\
\hline $\mathrm{H}$ & -1.829210 & 0.796116 & 0.817877 \\
\hline $\mathrm{H}$ & -1.829210 & 0.796116 & -0.817877 \\
\hline
\end{tabular}


$3^{+} \mathrm{C}$

\begin{tabular}{|c|c|c|c|}
\hline Atom & $\begin{array}{l}\text { Coorc } \\
\mathrm{X}\end{array}$ & $\begin{array}{c}\text { lates (Angs } \\
\text { Y }\end{array}$ & (oms) \\
\hline $\mathrm{N}$ & 0.000000 & 1.329304 & 0.000000 \\
\hline C & -0.090137 & -1.453575 & 0.000000 \\
\hline $\mathrm{C}$ & -1.373493 & -0.833659 & 0.000000 \\
\hline $\mathrm{C}$ & -1.309109 & 0.518234 & 0.000000 \\
\hline $\mathrm{C}$ & 1.243835 & 0.578571 & 0.000000 \\
\hline C & 1.184031 & -0.771555 & 0.000000 \\
\hline 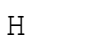 & -2.139680 & 1.234223 & 0.000000 \\
\hline 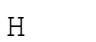 & 2.118996 & -1.337671 & 0.000000 \\
\hline $\mathrm{H}$ & -0.035086 & -2.551555 & 0.000000 \\
\hline & 2.155470 & 1.179419 & 0.000000 \\
\hline & -0.015232 & 1.971176 & 0.817466 \\
\hline $\mathrm{H}$ & -0.015232 & 1.971176 & -0.817466 \\
\hline
\end{tabular}

\section{$4 a$}

\begin{tabular}{|c|c|c|c|}
\hline Atom & $\begin{array}{l}\text { Coorc } \\
\mathrm{X}\end{array}$ & $\begin{array}{c}\text { ates (Angs } \\
Y\end{array}$ & oms ) \\
\hline 0 & 1.126203 & 0.703053 & 0.128124 \\
\hline $\mathrm{C}$ & -0.071366 & 1.284330 & 0.006559 \\
\hline $\mathrm{C}$ & -1.230108 & 0.567287 & -0.148193 \\
\hline $\mathrm{C}$ & -1.138047 & -0.833769 & 0.085873 \\
\hline $\mathrm{C}$ & 0.092728 & -1.481648 & -0.009573 \\
\hline $\mathrm{C}$ & 1.181218 & -0.667587 & -0.095394 \\
\hline $\mathrm{H}$ & -0.016459 & 2.368846 & 0.108514 \\
\hline $\mathrm{H}$ & -2.174922 & 1.103364 & -0.265860 \\
\hline $\mathrm{H}$ & -2.039510 & -1.367146 & 0.415629 \\
\hline . & 2.214713 & -0.941165 & -0.318903 \\
\hline
\end{tabular}

$4 b$

\begin{tabular}{|c|c|c|c|}
\hline Atom & $\begin{array}{l}\text { Coord } \\
x\end{array}$ & $\begin{array}{c}\text { rates (Angs } \\
\text { Y }\end{array}$ & ms ) \\
\hline 0 & -1.218123 & 0.641974 & 0.000000 \\
\hline $\mathrm{C}$ & 0.000000 & 1.280289 & 0.000000 \\
\hline $\mathrm{C}$ & 1.200606 & 0.636957 & 0.000000 \\
\hline $\mathrm{C}$ & 1.267602 & -0.797612 & 0.000000 \\
\hline $\mathrm{C}$ & 0.007268 & -1.393356 & 0.000000 \\
\hline 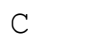 & -1.186709 & -0.760856 & 0.000000 \\
\hline $\mathrm{H}$ & -0.124097 & 2.362178 & 0.000000 \\
\hline $\mathrm{H}$ & 2.111307 & 1.240609 & 0.000000 \\
\hline & 2.210797 & -1.341643 & 0.000000 \\
\hline & -2.185621 & -1.189474 & 0.000000 \\
\hline
\end{tabular}




\section{$4 c$}

\begin{tabular}{|c|c|c|c|}
\hline Atom & \multicolumn{2}{|c|}{$\begin{array}{l}\text { Coordinates } \\
\mathrm{X}\end{array}$} & ms ) \\
\hline 0 & -1.150974 & 0.656792 & 0.000000 \\
\hline 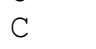 & 0.000000 & 1.313690 & 0.000000 \\
\hline $\mathrm{C}$ & 1.198373 & 0.639030 & 0.000000 \\
\hline $\mathrm{C}$ & 1.169037 & -0.770912 & 0.000000 \\
\hline $\mathrm{C}$ & -0.023543 & -1.544358 & 0.000000 \\
\hline $\mathrm{C}$ & -1.136290 & -0.726070 & 0.000000 \\
\hline $\mathrm{H}$ & -0.127704 & 2.397163 & 0.000000 \\
\hline $\mathrm{H}$ & 2.129031 & 1.212519 & 0.000000 \\
\hline $\mathrm{H}$ & 2.142377 & -1.282881 & 0.000000 \\
\hline $\mathrm{H}$ & -2.181378 & -1.049422 & 0.000000 \\
\hline
\end{tabular}

\section{$5 a$}

\begin{tabular}{|c|c|c|c|}
\hline Atom & $\begin{array}{l}\text { Coorc } \\
x\end{array}$ & $\begin{array}{c}\text { ates (Angs } \\
Y\end{array}$ & coms) \\
\hline & -1.278952 & -0.633073 & -0.095899 \\
\hline 0 & -1.103725 & 1.370032 & -0.023662 \\
\hline C & 0.491131 & -1.422722 & 0.041188 \\
\hline C & 1.569607 & -0.578197 & 0.092712 \\
\hline & 1.439131 & 0.871243 & -0.054903 \\
\hline & 0.196880 & 1.359215 & 0.049688 \\
\hline F & -1.787483 & 1.891982 & 0.658293 \\
\hline $\mathrm{F}$ & 0.706079 & -2.496554 & 0.089831 \\
\hline & 2.595976 & -0.966125 & 0.140005 \\
\hline & 2.261009 & 1.461067 & -0.466094 \\
\hline & -2.707350 & -1.257851 & 0.194512 \\
\hline
\end{tabular}

\section{$5 b$}

\begin{tabular}{|c|c|c|c|}
\hline Atom & $\begin{array}{l}\text { Coorc } \\
\mathrm{X}\end{array}$ & $\begin{array}{c}\text { ates } \\
\text { Y Ang }\end{array}$ & oms ) \\
\hline Al & 0.000000 & 1.485188 & 0.000000 \\
\hline 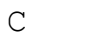 & 1.621396 & 0.374604 & 0.000000 \\
\hline $\mathrm{C}$ & -1.494560 & 0.223636 & 0.000000 \\
\hline $\mathrm{C}$ & -1.215915 & -1.130389 & 0.000000 \\
\hline $\mathrm{C}$ & 0.102638 & -1.686708 & 0.000000 \\
\hline $\mathrm{C}$ & 1.290965 & -0.932957 & 0.000000 \\
\hline $\mathrm{H}$ & 2.675495 & 0.684141 & 0.000000 \\
\hline $\mathrm{H}$ & -2.558716 & 0.491409 & 0.000000 \\
\hline $\mathrm{H}$ & -2.034281 & -1.863571 & 0.000000 \\
\hline $\mathrm{H}$ & 0.187887 & -2.779138 & 0.000000 \\
\hline 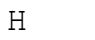 & -0.097531 & 3.070607 & 0.000000 \\
\hline
\end{tabular}


$5 c$

\begin{tabular}{|c|c|c|c|}
\hline Atom & $\begin{array}{l}\text { Coord } \\
x\end{array}$ & $\begin{array}{c}\text { rates (Angs } \\
\text { Y }\end{array}$ & oms ) \\
\hline Al & -1.154515 & -0.832017 & 0.000000 \\
\hline $\mathrm{C}$ & -1.265311 & 1.253958 & 0.000000 \\
\hline $\mathrm{C}$ & 0.665777 & -1.358942 & 0.000000 \\
\hline $\mathrm{C}$ & 1.619654 & -0.350297 & 0.000000 \\
\hline $\mathrm{C}$ & 1.326768 & 1.047880 & 0.000000 \\
\hline $\mathrm{C}$ & 0.000000 & 1.362950 & 0.000000 \\
\hline $\mathrm{H}$ & -2.264871 & 1.658223 & 0.000000 \\
\hline $\mathrm{H}$ & 1.036688 & -2.388412 & 0.000000 \\
\hline $\mathrm{H}$ & 2.688629 & -0.600838 & 0.000000 \\
\hline $\mathrm{H}$ & 2.094993 & 1.818495 & 0.000000 \\
\hline $\mathrm{H}$ & -2.628068 & -1.404535 & 0.000000 \\
\hline
\end{tabular}

\section{$6 a$}

\begin{tabular}{|c|c|c|c|}
\hline om & $\begin{array}{l}\text { Coordinates } \\
\text { X }\end{array}$ & $\begin{array}{c}\text { hates (Angs } \\
\text { Y }\end{array}$ & Z \\
\hline $\mathrm{Si}$ & 1.313493 & -0.360085 & 0.169944 \\
\hline & -0.265793 & -1.384836 & -0.179836 \\
\hline e & 0.764652 & 1.416428 & -0.237054 \\
\hline $\mathrm{C}$ & -0.533138 & 1.350794 & -0.019286 \\
\hline $\mathrm{C}$ & -1.613824 & 0.627671 & 0.236439 \\
\hline $\mathrm{C}$ & -1.486485 & -0.801121 & -0.174253 \\
\hline $\mathrm{H}$ & -2.401477 & -1.379064 & -0.348775 \\
\hline $\mathrm{H}$ & 1.842124 & -0.636934 & 1.546714 \\
\hline $\mathrm{H}$ & 2.376770 & -0.701606 & -0.828549 \\
\hline $\mathrm{H}$ & -0.198911 & -2.459151 & -0.388741 \\
\hline 11 & -2.418321 & 0.935936 & 0.908590 \\
\hline & 1.218444 & 2.028397 & -1.024520 \\
\hline
\end{tabular}

\section{$6 b$}

\begin{tabular}{|c|c|c|c|}
\hline Atom & $\begin{array}{l}\text { Coorc } \\
\mathrm{X}\end{array}$ & $\begin{array}{c}\text { nates (Ang } \\
\mathrm{Y}\end{array}$ & (oms) \\
\hline $\mathrm{Si}$ & 0.000000 & 1.411568 & 0.000000 \\
\hline $\mathrm{C}$ & 1.417524 & 0.180078 & 0.000000 \\
\hline $\mathrm{C}$ & -1.537729 & 0.310954 & 0.000000 \\
\hline $\mathrm{C}$ & -1.278986 & -1.012433 & 0.000000 \\
\hline $\mathrm{C}$ & -0.105034 & -1.780132 & 0.000000 \\
\hline $\mathrm{C}$ & 1.193275 & -1.177515 & 0.000000 \\
\hline $\mathrm{H}$ & 2.047501 & -1.865565 & 0.000000 \\
\hline $\mathrm{H}$ & 0.049069 & 2.312527 & 1.202732 \\
\hline $\mathrm{H}$ & 0.049069 & 2.312527 & -1.202732 \\
\hline $\mathrm{H}$ & 2.457709 & 0.524047 & 0.000000 \\
\hline $\mathrm{H}$ & -0.174635 & -2.871739 & 0.000000 \\
\hline T & -2.563017 & 0.700534 & 0.000000 \\
\hline
\end{tabular}




\begin{tabular}{|c|c|c|c|}
\hline Atom & $\begin{array}{c}\text { Coorc } \\
\mathrm{X}\end{array}$ & Coordinates (Angstroms) & coms) \\
\hline i & 0.000000 & 1.383889 & 0.000000 \\
\hline & 1.405101 & 0.182791 & 0.000000 \\
\hline C & -1.653143 & 0.266493 & 0.000000 \\
\hline C & -1.083625 & -0.884604 & 0.000000 \\
\hline C & -0.096815 & -1.810841 & 0.000000 \\
\hline $\mathrm{C}$ & 1.199181 & -1.174361 & 0.000000 \\
\hline $\mathrm{H}$ & 2.055599 & -1.859126 & 0.000000 \\
\hline $\mathrm{H}$ & -0.123641 & 2.289855 & 1.198180 \\
\hline $\mathrm{H}$ & -0.123641 & 2.289855 & -1.198180 \\
\hline $\mathrm{H}$ & 2.435314 & 0.550552 & 0.000000 \\
\hline $\mathrm{H}$ & -0.254066 & -2.884821 & 0.000000 \\
\hline & -2.613762 & 0.762364 & 0.000000 \\
\hline
\end{tabular}

\section{$6 d$}

\begin{tabular}{|c|c|c|c|}
\hline Atom & $\begin{array}{l}\text { Coor } \\
\mathrm{X}\end{array}$ & $\begin{array}{c}\text { hates (Angs } \\
\text { Y }\end{array}$ & coms) \\
\hline $\mathrm{Si}$ & 0.000000 & 1.398983 & 0.000000 \\
\hline $\mathrm{C}$ & -1.443902 & -1.156570 & 0.000000 \\
\hline C & -1.498556 & 0.243289 & 0.000000 \\
\hline $\mathrm{C}$ & 1.475917 & 0.235533 & 0.000000 \\
\hline $\mathrm{C}$ & 1.220996 & -1.102094 & 0.000000 \\
\hline C & -0.114192 & -1.689789 & 0.000000 \\
\hline $\mathrm{H}$ & 2.055591 & -1.815550 & 0.000000 \\
\hline 11 & 0.071334 & 2.275109 & 1.216621 \\
\hline $\mathrm{H}$ & 0.071334 & 2.275109 & -1.216621 \\
\hline $\mathrm{H}$ & 2.517315 & 0.576070 & 0.000000 \\
\hline $\mathrm{H}$ & -0.060278 & -2.792389 & 0.000000 \\
\hline $\mathrm{H}$ & -2.496877 & 0.713668 & 0.000000 \\
\hline
\end{tabular}

$7 a$

\begin{tabular}{|c|c|c|c|}
\hline Atom & \multicolumn{2}{|c|}{$\begin{array}{l}\text { Coordinates } \\
\text { X }\end{array}$} & coms ) \\
\hline $\mathrm{P}$ & -1.382332 & -0.320473 & 0.049974 \\
\hline $\mathrm{C}$ & 0.144097 & -1.336843 & -0.107595 \\
\hline $\mathrm{C}$ & -0.690477 & 1.381052 & -0.179198 \\
\hline $\mathrm{C}$ & 0.617935 & 1.345369 & 0.006585 \\
\hline $\mathrm{C}$ & 1.660748 & 0.540136 & 0.208797 \\
\hline $\mathrm{C}$ & 1.412521 & -0.852562 & -0.186403 \\
\hline $\mathrm{H}$ & -1.673823 & -0.563052 & 1.440283 \\
\hline $\mathrm{H}$ & -1.213146 & 2.055252 & -0.862869 \\
\hline $\mathrm{H}$ & 2.530604 & 0.794444 & 0.819735 \\
\hline $\mathrm{H}$ & 2.252213 & -1.529111 & -0.379776 \\
\hline $\mathrm{H}$ & -0.029808 & -2.413345 & -0.220094 \\
\hline
\end{tabular}


$7 b$

\begin{tabular}{|c|c|c|c|}
\hline Atom & \multicolumn{3}{|c|}{ Coordinates (Angstroms) } \\
\hline$P$ & 1.408924 & -0.233207 & -0.097299 \\
\hline $\mathrm{C}$ & -0.782454 & 1.403788 & 0.029010 \\
\hline $\mathrm{C}$ & 0.566094 & 1.395895 & -0.016917 \\
\hline $\mathrm{C}$ & -1.740325 & 0.391159 & 0.029221 \\
\hline $\mathrm{C}$ & -1.320556 & -0.968172 & -0.028278 \\
\hline $\mathrm{C}$ & -0.001705 & -1.360882 & 0.018906 \\
\hline $\mathrm{H}$ & 1.185627 & 2.297389 & -0.009221 \\
\hline $\mathrm{H}$ & -2.806298 & 0.626265 & 0.072823 \\
\hline $\mathrm{H}$ & -2.097095 & -1.741239 & -0.048583 \\
\hline $\mathrm{H}$ & 0.238332 & -2.428099 & 0.047791 \\
\hline $\mathrm{H}$ & 2.019249 & -0.426942 & 1.205027 \\
\hline
\end{tabular}

7c

\begin{tabular}{|c|c|c|c|}
\hline Atom & $\begin{array}{l}\text { Coord } \\
x\end{array}$ & $\begin{array}{c}\text { ates (An } \\
\text { Y }\end{array}$ & roms) \\
\hline $\mathrm{P}$ & 1.363679 & -0.162994 & -0.100821 \\
\hline & -0.864856 & 1.405905 & 0.052227 \\
\hline & 0.477000 & 1.456644 & 0.011186 \\
\hline C & -1.715659 & 0.293004 & 0.022742 \\
\hline C & -1.250838 & -1.033200 & -0.060502 \\
\hline $\mathrm{C}$ & 0.097345 & -1.360223 & 0.051640 \\
\hline 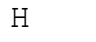 & 1.116882 & 2.341070 & 0.035783 \\
\hline $\mathrm{H}$ & -2.800295 & 0.445279 & 0.065850 \\
\hline $\mathrm{H}$ & -1.985977 & -1.845888 & -0.083724 \\
\hline $\mathrm{H}$ & 0.391200 & -2.401460 & 0.206806 \\
\hline 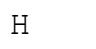 & 2.365045 & -0.666877 & 0.823850 \\
\hline
\end{tabular}

\section{$8 a$}

\begin{tabular}{|c|c|c|c|}
\hline Atom & $\begin{array}{l}\text { Coorc } \\
\mathrm{X}\end{array}$ & $\begin{array}{c}\text { ates (Angs } \\
Y\end{array}$ & oms ) \\
\hline$P$ & 1.580208 & -0.576530 & 0.072437 \\
\hline$P$ & -1.662929 & -0.526837 & -0.161086 \\
\hline $\mathrm{C}$ & 0.026117 & 1.521878 & 0.009036 \\
\hline $\mathrm{C}$ & 1.268183 & 1.208168 & -0.299185 \\
\hline $\mathrm{C}$ & -1.219600 & 1.221346 & 0.302659 \\
\hline $\mathrm{C}$ & -0.136285 & -1.257613 & -0.011408 \\
\hline $\mathrm{H}$ & 1.856729 & 1.680574 & -1.089093 \\
\hline $\mathrm{H}$ & -1.858795 & 1.752262 & 1.012481 \\
\hline $\mathrm{H}$ & -0.150804 & -2.354888 & -0.094847 \\
\hline $\mathrm{H}$ & 1.763190 & -0.690116 & 1.494594 \\
\hline
\end{tabular}


$8 b$

\begin{tabular}{|c|c|c|c|}
\hline Atom & $\begin{array}{l}\text { Coord } \\
X\end{array}$ & $\begin{array}{c}\text { hates (Angs } \\
\text { Y }\end{array}$ & coms) \\
\hline$P$ & -1.632753 & -0.597520 & -0.033753 \\
\hline $\mathrm{P}$ & 1.565580 & -0.551904 & -0.110969 \\
\hline $\mathrm{C}$ & -0.061752 & -1.289294 & 0.067649 \\
\hline $\mathrm{C}$ & -1.311307 & 1.158842 & 0.043012 \\
\hline C & -0.038493 & 1.690098 & 0.037041 \\
\hline $\mathrm{C}$ & 1.235397 & 1.244997 & -0.016067 \\
\hline $\mathrm{H}$ & 2.102945 & 1. 912124 & -0.005435 \\
\hline $\mathrm{H}$ & -2.189779 & 1.811070 & 0.096296 \\
\hline $\mathrm{H}$ & -0.045036 & -2.383608 & 0.152129 \\
\hline I & 2.196400 & -0.926084 & 1.138032 \\
\hline
\end{tabular}

\section{$8 c$}

\begin{tabular}{|c|c|c|c|}
\hline Atom & \multicolumn{3}{|c|}{ Coordinates (Angstroms) } \\
\hline$P$ & 1.557997 & -0.539513 & -0.126558 \\
\hline $\mathrm{P}$ & -1.622745 & -0.608894 & -0.057888 \\
\hline $\mathrm{C}$ & -0.054387 & 1.650249 & 0.057242 \\
\hline $\mathrm{C}$ & 1.220857 & 1.321394 & 0.005820 \\
\hline $\mathrm{C}$ & -1.326781 & 1.141099 & 0.052481 \\
\hline $\mathrm{C}$ & -0.035082 & -1.271436 & 0.101791 \\
\hline $\mathrm{H}$ & 2.110415 & 1.950537 & 0.027436 \\
\hline $\mathrm{H}$ & -2.213955 & 1.779454 & 0.115683 \\
\hline $\mathrm{H}$ & -0.013528 & -2.337095 & 0.361012 \\
\hline $\mathrm{H}$ & 2.260650 & -1.214617 & 0.958553 \\
\hline
\end{tabular}

$9 a$

\begin{tabular}{|c|c|c|c|}
\hline Atom & $\begin{array}{l}\text { Coord } \\
x\end{array}$ & $\begin{array}{c}\text { hates (Angs } \\
\text { Y }\end{array}$ & coms ) \\
\hline S & 1.368061 & -0.237797 & -0.164998 \\
\hline C & -0.000372 & -1.307361 & 0.118495 \\
\hline $\mathrm{C}$ & -1.306878 & -0.924684 & 0.202330 \\
\hline C & -1.661311 & 0.421224 & -0.215824 \\
\hline $\mathrm{C}$ & -0.733966 & 1.380674 & -0.003013 \\
\hline $\mathrm{C}$ & 0.581814 & 1.304528 & 0.241247 \\
\hline $\mathrm{H}$ & -2.065350 & -1.684130 & 0.413123 \\
\hline $\mathrm{H}$ & 1.186948 & 2.017636 & 0.806637 \\
\hline $\mathrm{H}$ & -2.577905 & 0.585133 & -0.792432 \\
\hline $\mathrm{H}$ & 0.291614 & -2.360161 & 0.153238 \\
\hline
\end{tabular}


$9 b$

\begin{tabular}{|c|c|c|c|}
\hline Atom & $\begin{array}{l}\text { Coord } \\
X\end{array}$ & $\begin{array}{r}\text { nates (An } \\
\text { Y }\end{array}$ & Z \\
\hline S & -1.404742 & 0.263680 & 0.000000 \\
\hline $\mathrm{C}$ & 0.000000 & 1.333705 & 0.000000 \\
\hline $\mathrm{C}$ & 1.309955 & 0.933658 & 0.000000 \\
\hline $\mathrm{C}$ & 1.736922 & -0.428040 & 0.000000 \\
\hline $\mathrm{C}$ & 0.746182 & -1.404350 & 0.000000 \\
\hline $\mathrm{C}$ & -0.598617 & -1.344668 & 0.000000 \\
\hline $\mathrm{H}$ & -0.276587 & 2.389413 & 0.000000 \\
\hline $\mathrm{H}$ & 2.072970 & 1.718781 & 0.000000 \\
\hline $\mathrm{H}$ & 2.798650 & -0.677132 & 0.000000 \\
\hline $\mathrm{H}$ & -1.285816 & -2.191780 & 0.000000 \\
\hline
\end{tabular}

$9 c$

\begin{tabular}{|c|c|c|c|}
\hline tom & \multicolumn{3}{|c|}{ Coordinates (Angstroms) } \\
\hline $\mathrm{S}$ & -1.354605 & 0.328720 & 0.000000 \\
\hline $\mathrm{C}$ & -0.600550 & -1.273751 & 0.000000 \\
\hline $\mathrm{C}$ & 0.000000 & 1.358671 & 0.000000 \\
\hline $\mathrm{C}$ & 1.300570 & 0.887454 & 0.000000 \\
\hline $\mathrm{C}$ & 1.634711 & -0.481762 & 0.000000 \\
\hline $\mathrm{C}$ & 0.746750 & -1.590808 & 0.000000 \\
\hline $\mathrm{H}$ & 2.101022 & 1.634424 & 0.000000 \\
\hline $\mathrm{H}$ & -1.400592 & -2.025060 & 0.000000 \\
\hline $\mathrm{H}$ & 2.713848 & -0.694018 & 0.000000 \\
\hline $\mathrm{H}$ & -0.229476 & 2.426311 & 0.000000 \\
\hline
\end{tabular}

